\title{
Gestión de base de datos con SCADA para el control automatizado de una válvula de control proporcional
}

\begin{abstract}
RESUMEN
El artículo analiza la importancia y aplicaciones de la gestión de base de datos en los procesos automatizados, con el objetivo de realizar un buen control de la producción y los recursos humanos, mantenimiento de los dispositivos, procesamiento de información para una adecuada toma de decisiones en la empresa y la mejora de los procesos industriales. Para mostrar la interacción software-hardware se han diseñado aplicaciones con el Software Intouch durante el "I Curso Internacional de Supervisión y Automatización totalmente integrada" (SENA-Colombia), SQL Server de Microsoft en el Centro de Manufactura Avanzada (CEMAUNMSM) y LabVIEW de National Instruments en la empresa Equipment \& Systems for Advanced Technology SAC (E-SAT) y con hardware diferente.

Palabras clave: SCADA, instrumentación, base de datos, DBMS
\end{abstract}

BASE MANAGEMENT OF INFORMATION WITH SCADA FOR THE AUTOMATED CONTROL OF A VALVE OF PROPORTIONAL CONTROL

\section{ABSTRACT}

This article discusses the importance and applications of the Database Management in Automated Processes, with the objective of to make a good control of the production and human resources, maintenance of the devices, processing of information for an adapted making decisions and the improvement of the Industrial Proccesses. To show the interaction software-hardware have been designed applications with Intouch Software during "I Curso Internacional de Supervisión y Automatización totalmente integrada" (SENA-Colombia), Microsoft SQL Server at the Centro de Manufactura Avanzada (CEMA-UNMSM) and LABVIEW in the company Equipment \& Systems for Advanced Technology SAC (E-SAT PERU) and different hardware.

Keywords: SCADA, instrumentation, database, DBMS

\section{INTRODUCCIÓN}

La automatización industrial es una disciplina que involucra principalmente a las especialidades de la ingeniería electrónica, industrial, química, mecánica, mecatrónica y de sistemas que va desde un sencillo "sistema de control"; hasta la instrumentación industrial, sistemas de control y supervisión, los sistemas de transmisión y recolección de datos y las aplicaciones de software en tiempo real para supervisar y controlar las operaciones de procesos y plantas industriales.

Las aplicaciones de software en tiempo real permiten administrar el control de procesos para mantener estable un sistema automatizado de producción industrial; sin embargo, también es vital que los datos que son suministrados por el sistema (VP: Variables de Proceso) y las variables de referencia (SP: Set Point) y otras deben almacenarse adecuadamente en una base de datos para su posterior análisis permitiendo realizar toma de decisiones adecuadas tanto a nivel gerencial como decisiones en una planta industrial a nivel correctivo, preventivo y predictivo.

\section{DEFINICIONES}

Instrumentación Industrial: Conocimiento de la correcta aplicación de los equipos encaminados a apoyar al operario/analista de procesos en la medición, regulación, observación, seguridad y almacenamiento del valor de una variable dentro de un proceso productivo. Como parte de la instrumentación industrial podemos mencionar a los sensores, actuadores, controladores, tuberías, mandos eléctricos, suministros de energía entre otros.

Sistemas de Control: Conjunto de dispositivos (instrumentación industrial) adicionales al proceso, que llevan o ayudan al control o regulación del proceso [1]; permitiendo fabricar productos con mayor calidad a menor costo y tiempos óptimos de producción.

SCADA: Supervisory Control and Data Adquisition (Supervisión de Control y Adquisición de Datos): Aplicación de software

\footnotetext{
1 Jefe de Planes y Programas UNMSM. Investigador del Centro de Manufactura Avanzada CEMA - UNMSM

E-mail: jorgeepd@hotmail.com

2 Ingeniero Industrial UNMSM. Coordinador de la Empresa Southern Perú. E-mail: robertotelloyuen@gmail.com

3 Egresada de Ingeniería Industrial UNMSM. Investigadora del Centro de Manufactura Avanzada - CEMA UNMSM.

E-mail: libe.sm04@gmail.com
} 
especialmente diseñado para la captura de información de un proceso o planta industrial (aunque no es absolutamente necesario que pertenezca a este ámbito), con esta información es posible realizar una serie de análisis o estudios con los que se pueden obtener valiosos indicadores que permitan una retroalimentación sobre un operador o sobre el propio proceso.

El avance de la tecnología ha motivado a que las empresas apliquen las diferentes metodologías para automatizar y controlar sus procesos, aumentando su productividad y competitividad, constituyéndose en un factor clave para el éxito empresarial [2].

Base de Datos: Conjunto de datos que pertenecen al mismo contexto, almacenados sistemáticamente para su posterior uso; mientras que un sistema administrador de base de datos (DBMS) es una herramienta de software que permiten crear estructuras, almacenar datos y posteriormente acceder a ellos de forma rápida, segura y eficiente.

SQL: Structured Query Language (Lenguaje de Consulta Estructurado), conjunto de instrucciones normalizadas que permite trabajar con los objetos de una base de datos (colecciones, tablas, vistas, índices) y permiten realizar operaciones con los datos (consulta, ingreso, modificación y eliminación) [3].

\section{PLANTEAMIENTO DE LA APLICACIÓN}

La aplicación consiste en adquirir, supervisar y controlar el valor del caudal que circula por la válvula de control proporcional, la cual cambiará su porcentaje de apertura, guardando los valores en una Base de Datos con el objetivo de interactuar con la información y procesarla adecuadamente para la toma de decisiones.

\section{DIAGRAMA DE FLUJO DEL PROCESO}

Figura 1. Descripción del proceso de flujo a través de la válvula.

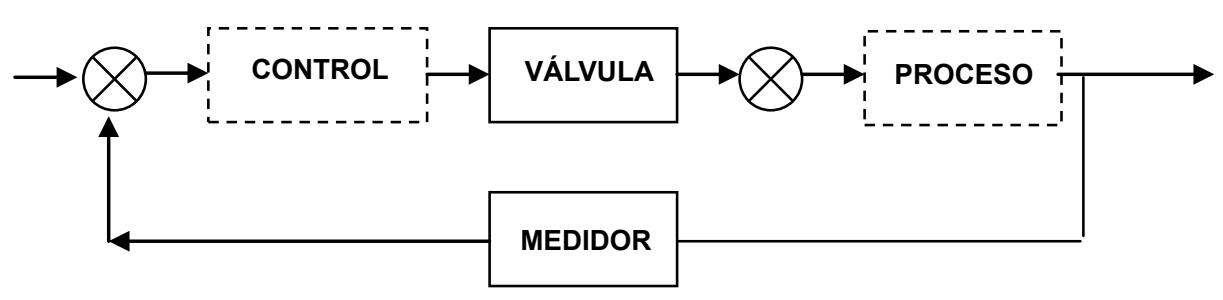

Fuente: Elaboración propia

\section{IMPLEMENTACIÓN EN INTOUCH}

Se muestra los pasos para establecer la comunicación entre la herramienta SCADA (Intouch); configurando el software de comunicaciones denominado RsLinx para acceder a los datos del PLC Allen Bradley.

Figura 2. Configuración de los módulos del Control (PLC)

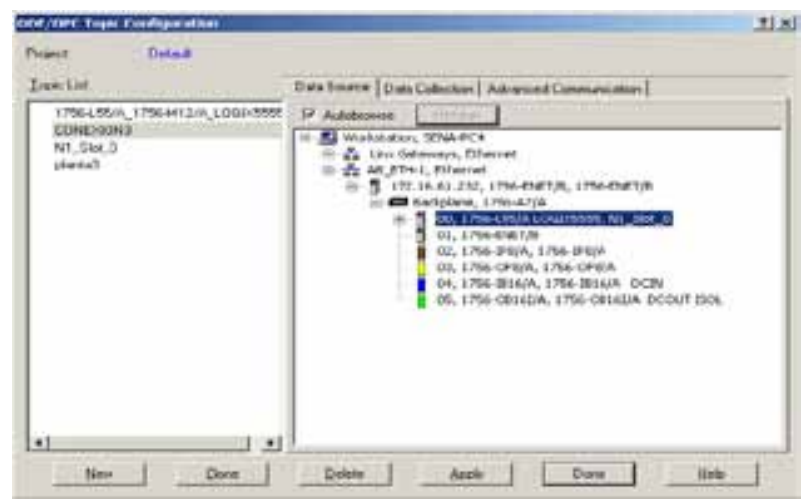

Fuente: Elaboración propia
Se importa el programa desarrollado en la herramienta de software "RSLogix5000" a la memoria del PLC (Allen Bradley),

Figura 3. Ventana principal del RSLogix5000

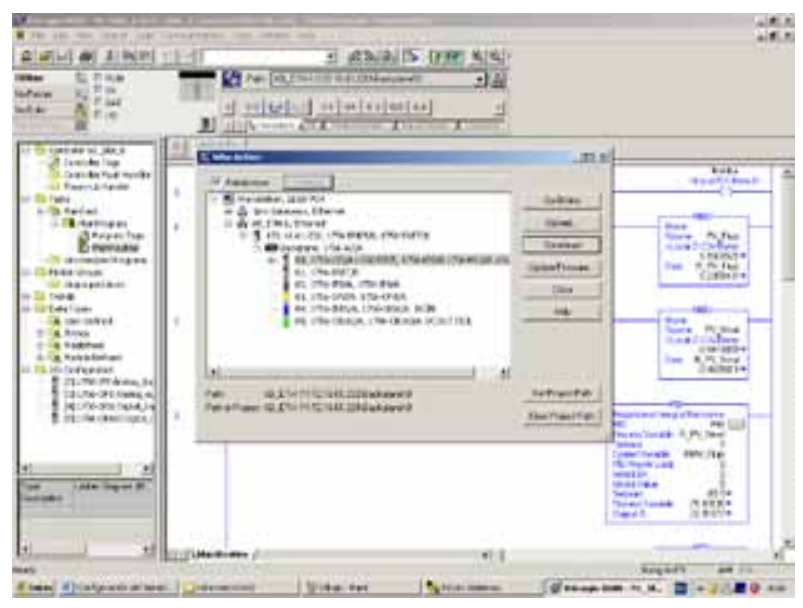

Fuente: Elaboración propia 
Se realiza la interfaz hombre-máquina para visualizar un dato de la planta de proceso (R_PV_Flujo: Variable de Proceso de Flujo) para lo cual se emplea el Window Maker de Intouch.

Figura 4. Pantalla inicial del Software SCADA Intouch

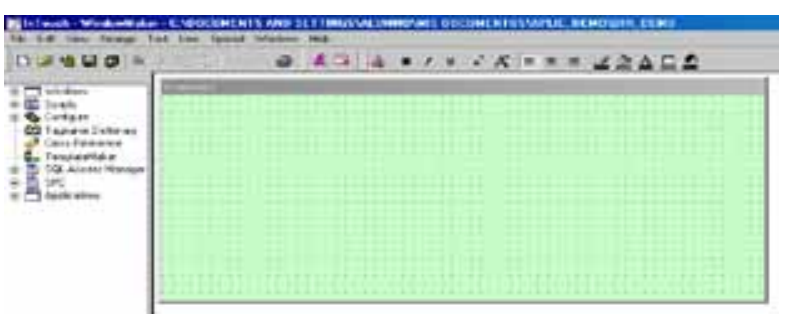

Fuente: Elaboración propia

Se realiza el esquemático de nuestro proceso real en Intouch, para lo cual insertamos los símbolos un tanque, tuberías, la válvula y la bomba.

Figura 5. Inserción de elementos en la Plataforma de Trabajo.

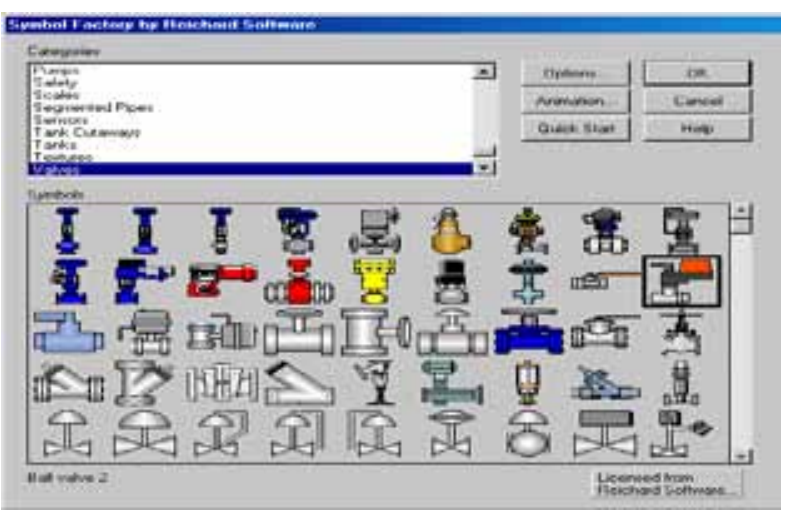

Fuente: Elaboración propia

Figura 6. Se acoplan los componentes insertados

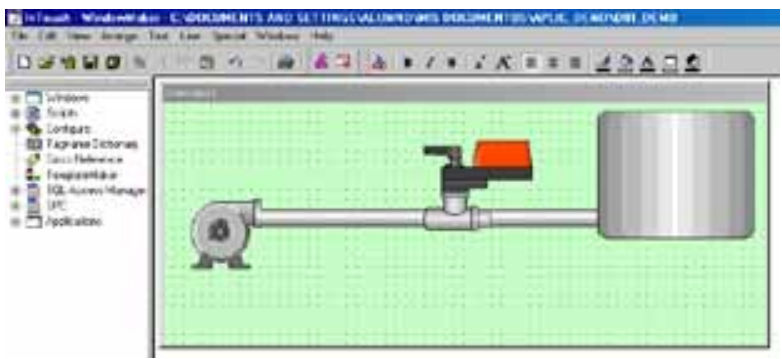

Fuente: Elaboración propia
Figura 7. Se configura el tipo de entradas

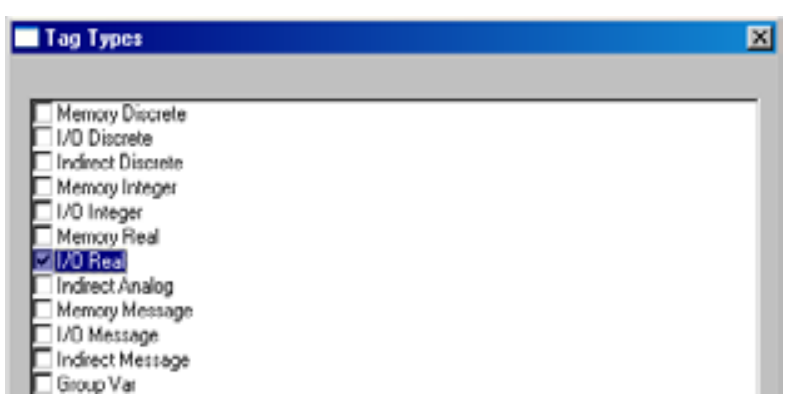

Fuente: Elaboración propia

Figura 8. Se configura el Protocolo de Comunicaciones

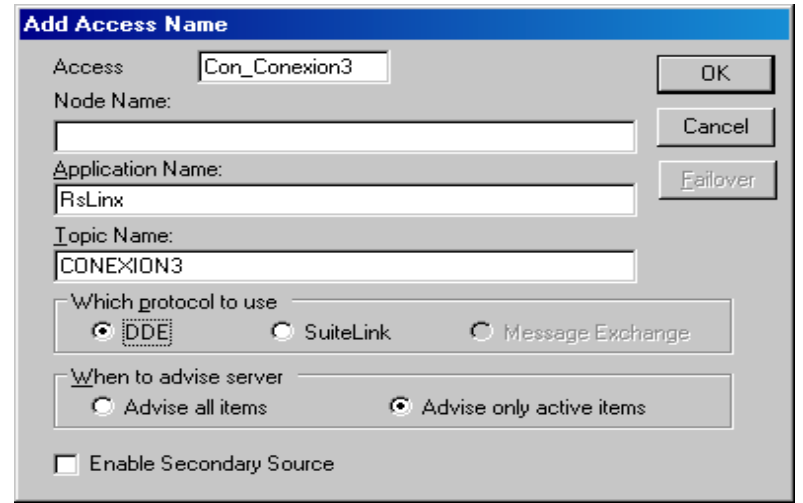

Fuente: Elaboración propia

Figura 9. Se configura las entradas analógicas

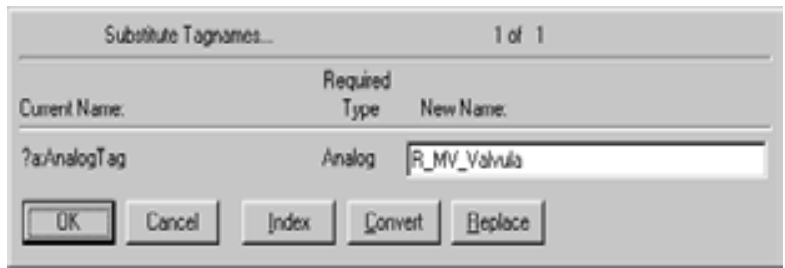

Fuente: Elaboración propia

Figura 10. Se establece la comunicación con la Base de Datos

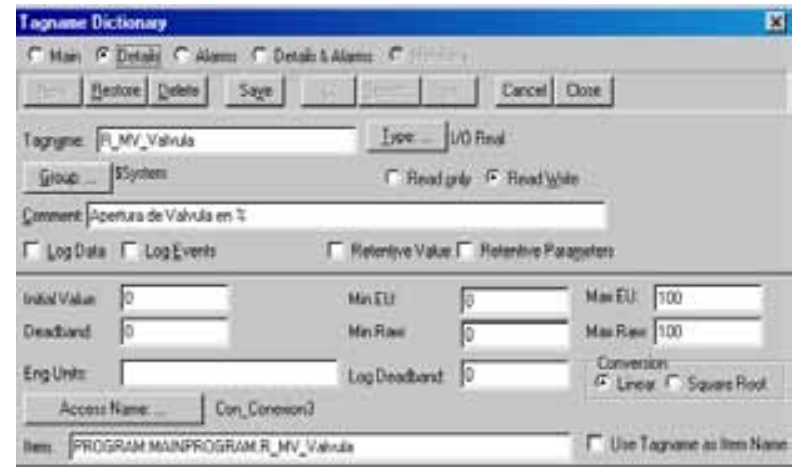

Fuente: Elaboración propia 
Figura 11. Accesar a la base de datos de la tabla creada en el SQL Server

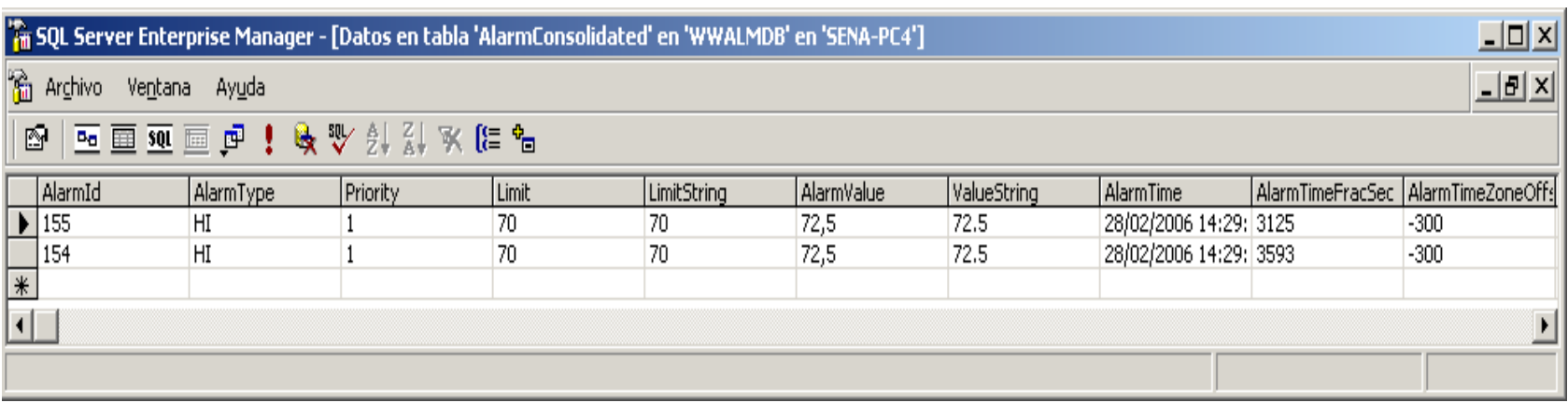

Fuente: Elaboración propia

Figura 12. Proceso en estado inicial cuyos datos serán registrados

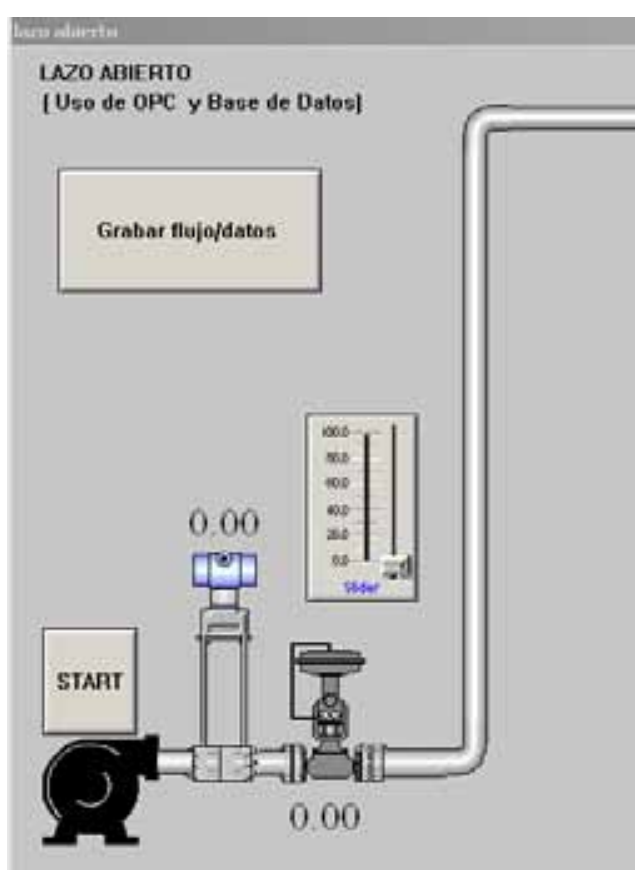

Fuente: Elaboración propia
Figura 13. Diseño de la tabla de la Base de Datos

\begin{tabular}{|c|c|c|c|c|c|c|}
\hline \multicolumn{5}{|c|}{ 通 Archivo Ventana Ayuda } & \multicolumn{2}{|c|}{ - } \\
\hline \multicolumn{7}{|c|}{ 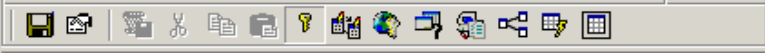 } \\
\hline \multicolumn{2}{|c|}{\begin{tabular}{|l|l} 
& Nombre de columna \\
\end{tabular}} & Tipo de datos & \multirow{2}{*}{$\frac{\text { Longitud }}{4}$} & \multicolumn{2}{|c|}{ Permitir valores nulos } & $\Delta$ \\
\hline \multirow{2}{*}{18} & AlarmId & int & & \multicolumn{2}{|c|}{ (l) } & \multirow[t]{3}{*}{-} \\
\hline & AlarmId & & 6 & & & \\
\hline \multicolumn{2}{|r|}{ Priority } & smallint & 2 & & & \\
\hline \multicolumn{2}{|r|}{ Limit } & float & 8 & \multicolumn{2}{|l|}{$\checkmark$} & \\
\hline \multicolumn{2}{|r|}{ LimitString } & nvarchar & 131 & \multicolumn{2}{|l|}{$\checkmark$} & \\
\hline \multicolumn{2}{|r|}{ AlarmValue } & float & 8 & \multicolumn{2}{|l|}{$\checkmark$} & \\
\hline \multicolumn{2}{|r|}{ ValueString } & nvarchar & 131 & $\checkmark$ & & \\
\hline & AlarmTime & datetime & 8 & & & \\
\hline & AlarmTimeFracSec & smallint & 2 & & & \\
\hline & AlarmTimeZoneoffset & smallint & 2 & & & \\
\hline & AlarmDaylightadjustm & smallint & 2 & & & \\
\hline & CommentId & int & 4 & $\checkmark$ & & \\
\hline & OutstandingAcks & smallint & 2 & $\checkmark$ & & \\
\hline & AckTime & datetime & 8 & $\checkmark$ & & \\
\hline & AckTimeFracSec & smallint & 2 & $\checkmark$ & & \\
\hline & AckTimeZoneOffset & smallint & 2 & $\checkmark$ & & \\
\hline & AckDaylightAdjustmer & smallint & 2 & $\checkmark$ & & \\
\hline & AckOperatorName & nvarchar & 131 & $\checkmark$ & & \\
\hline & AckNodeName & nvarchar & 32 & $\checkmark$ & & \\
\hline & AckCommentId & int & 4 & $\checkmark$ & & \\
\hline & ReturnValue & float & 8 & $\checkmark$ & & \\
\hline & ReturnValueString & nvarchar & 131 & $\checkmark$ & & \\
\hline & ReturnTime & datetime & 8 & $\checkmark$ & & \\
\hline & ReturnTimeFracSec & smallint & 2 & $\checkmark$ & & \\
\hline & ReturnTimeZoneoffse & smallint & 2 & $\checkmark$ & & \\
\hline & ReturnDaylightAdjustr & smallint & 2 & $\checkmark$ & & \\
\hline & User1 & float & 8 & $\checkmark$ & & \\
\hline & User2 & float & 8 & $\checkmark$ & & \\
\hline & User3 & nvarchar & 131 & $\checkmark$ & & \\
\hline & Cookie & int & 4 & $\checkmark$ & & \\
\hline & OperatorName & nvarchar & 131 & $\checkmark$ & & \\
\hline & OperatorNode & nvarchar & 32 & $\checkmark$ & & \\
\hline & UnAckDuration & char & 17 & $\checkmark$ & & \\
\hline & OperatorID & int & 4 & $\checkmark$ & & \\
\hline & AckOperatorID & int & 4 & $\checkmark$ & & - \\
\hline & Columnas & & & & & \\
\hline
\end{tabular}

Fuente: Elaboración propia 
Figura 14. Datos y gráfica realizados de manera experimental

\begin{tabular}{cc}
\hline Apertura (\%) & Flujo (\%) \\
\hline 0 & 0 \\
\hline 10 & 29,7672577 \\
\hline 15 & 40,3120308 \\
\hline 20 & 46,3656578 \\
\hline 25 & 51,8107796 \\
\hline 30 & 56,2037811 \\
\hline 35 & 59,4602547 \\
\hline 40 & 62,3496628 \\
\hline 45 & 64,6050568 \\
\hline 50 & 66,9880295 \\
\hline 55 & 69,3219376 \\
\hline 60 & 70,0599899 \\
\hline 65 & 69,4338226 \\
\hline 70 & 69,9834366 \\
\hline 75 & 70,9118958 \\
\hline 80 & 71,5832062 \\
\hline 85 & 72,4135284 \\
\hline 90 & 72,8630371 \\
\hline 95 & 73,3517914 \\
\hline 100 & 73,8562622 \\
\hline
\end{tabular}

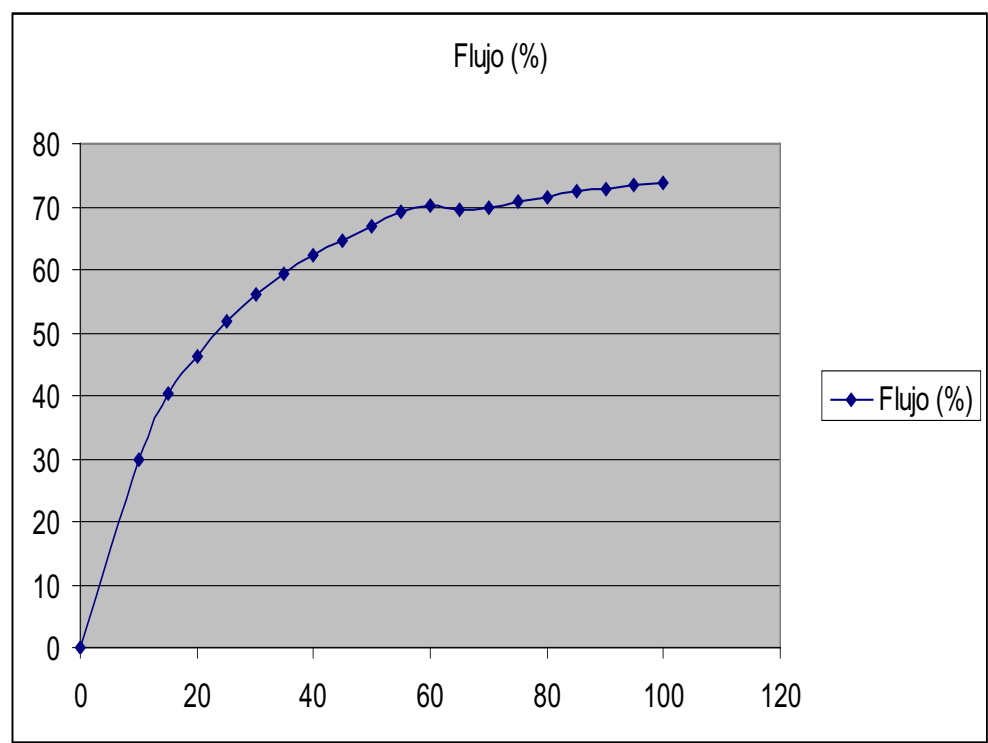

Fuente: Elaboración propia

El Intouch es una herramienta muy potente que permite la comunicación con una base de datos externa (SQL Server, Oracle, DB2, Informix, etc.) donde se puede grabar los valores de las variables (TAGs) de un HMI (Interfaz Hombre Máquina) en tiempo real; para luego, poder analizar esta información mediante un análisis estadístico (gráficas, histogramas, pastel, etc.) y así poder tomar decisiones correctas y oportunas del área de mantenimiento.

Se hace uso de un sistema de comunicación el cual nos sirve para un control ya sea eléctrico ó neumático, por medio del PLC y herramientas de software, el cual permite llevar un registro histórico del proceso industrial, para conocer las tendencias, comportamientos, tiempos de parada, mantenimiento predictivo y correctivo (Figura 15).

Se ejecuta la siguiente sentencia (Figura 16):

\section{SQL/> SELECT * FROM TABLA_FLUJO_APER- TURA}

Se ingresa al Software LABVIEW y se utiliza el Database Connectivity Toolkit, el cual tiene el siguiente Menú para Gestión de Base de Datos en el Diagrama de Bloques (Figura 17).
Posteriormente, se realiza la estructura mostrada en la Figura 18 en el Diagrama de Bloques, para guardar los datos en la ruta: C:le-satlRegistro

Finalmente, hacemos uso de SQL para visualizar la información ordenada en función del porcentaje de apertura (Figura 19).

Figura 15. Accediendo al Administrador de Consultas Microsoft SQL Server

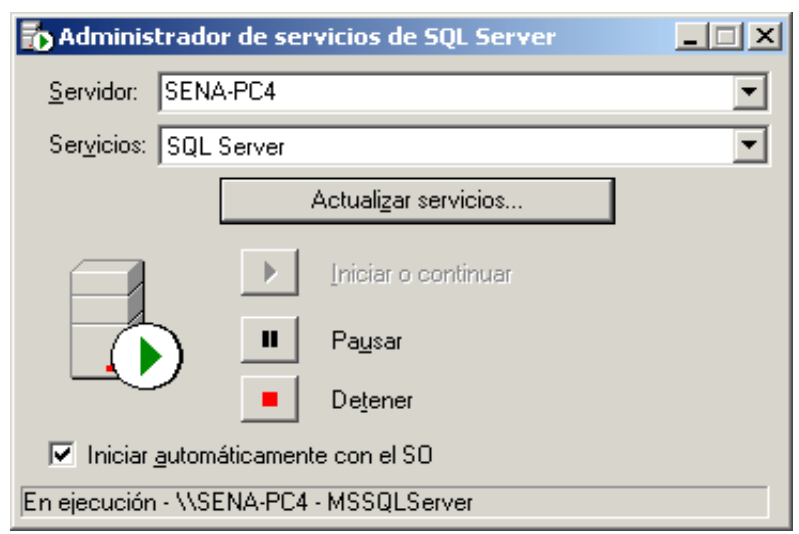

Fuente: Elaboración propia 
Figura 16. Visualización de los datos para la sentencia SQL realizada

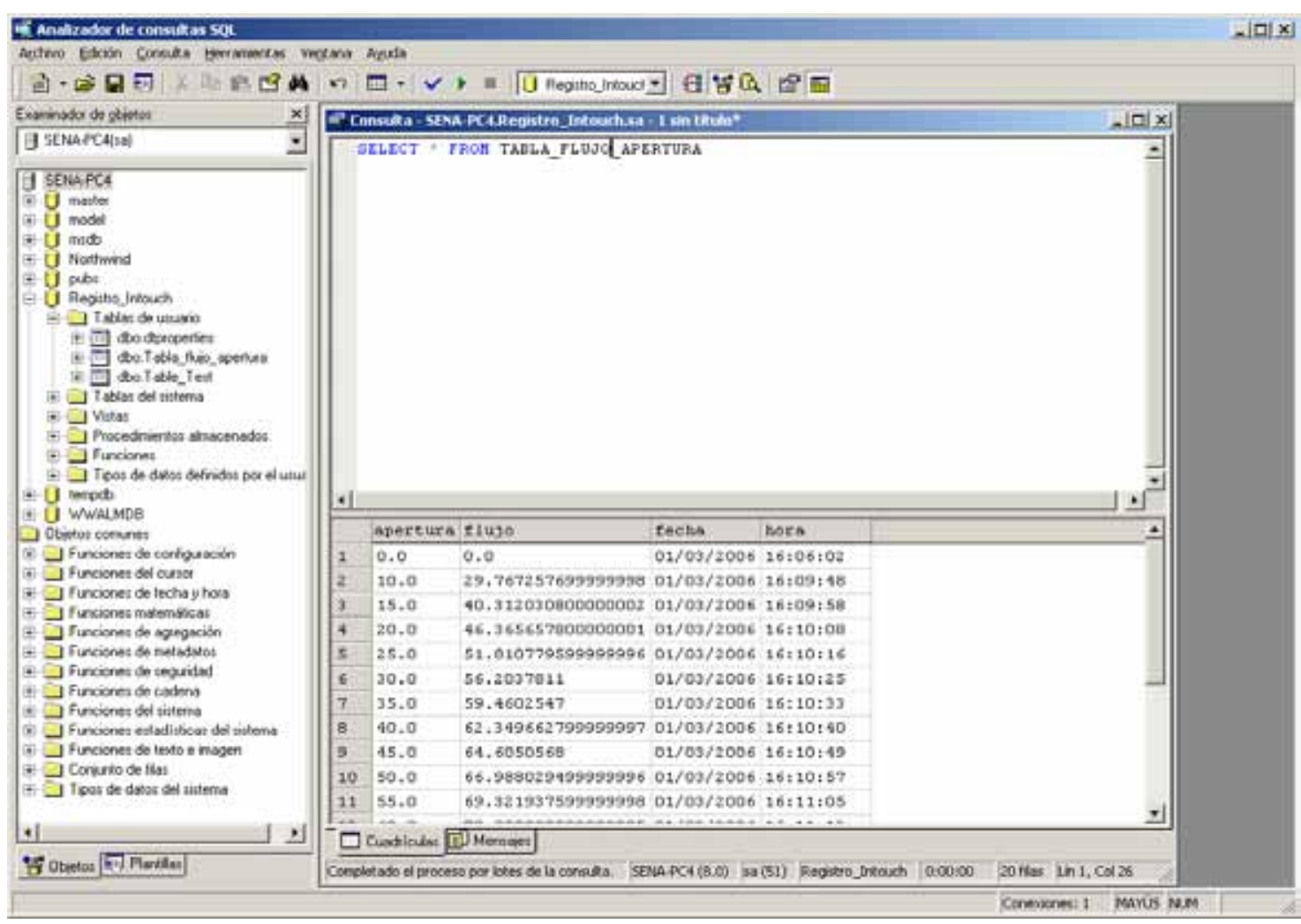

Fuente: Elaboración propia

Figura 17. Subpaleta del LABVIEW para la conexión de Base de Datos

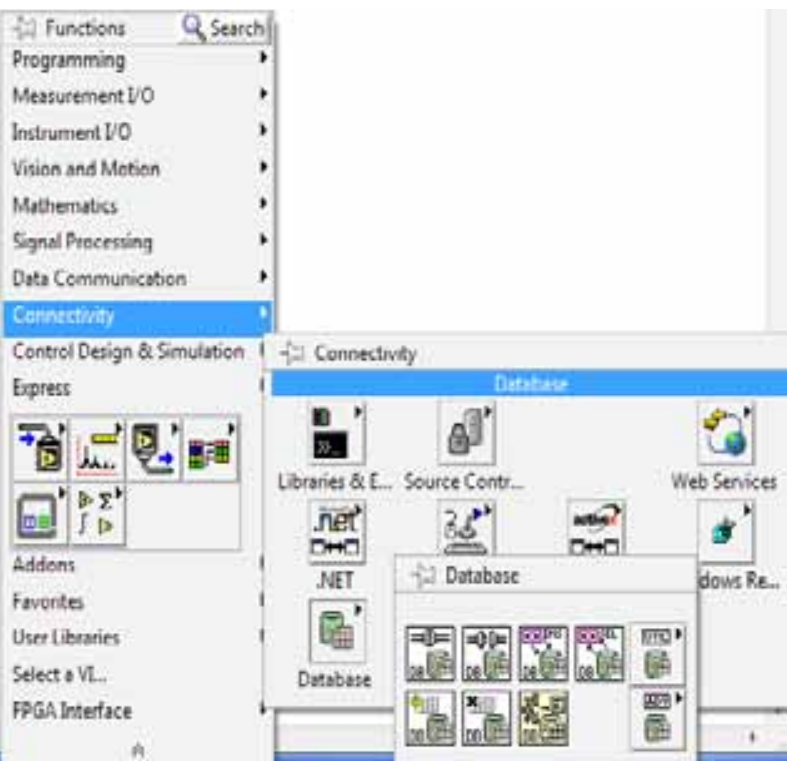

Fuente: Elaboración propia
Figura 18. Código fuente de LABVIEW requerido para guardar la Base de Datos en archivo.

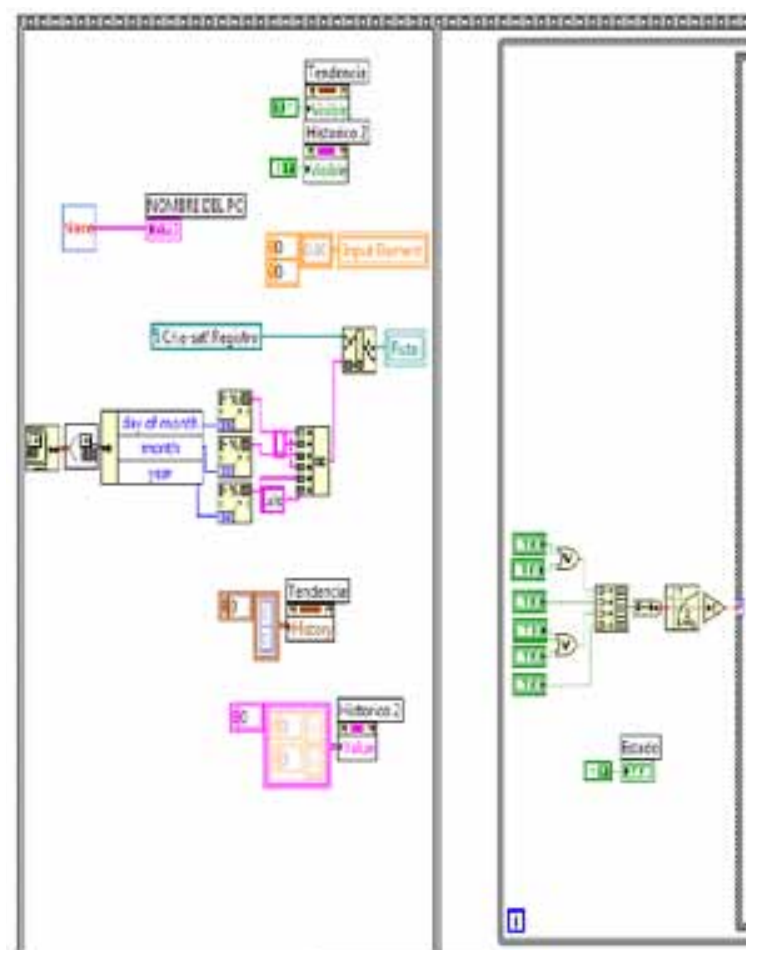

Fuente: Elaboración propia 
Figura 19. Sentencias SQL para consultar el flujo del proceso

$$
\begin{aligned}
& \text { SELECT * } \\
& \text { FROM TABLA_FLUJO_APERTURA } \\
& \text { WHERE APERTURA BETWEEN } 35 \text { AND } 80 \\
& \text { ORDER BY APERTURA }
\end{aligned}
$$

Fuente: Elaboración propia

Figura 20. Reporte del flujo del proceso vs. apertura de la válvula

\begin{tabular}{|cccc|}
\hline Resultado: & \multicolumn{3}{c|}{} \\
\hline APERTURA & \multicolumn{1}{c}{ FLUJO } & FECHA & HORA \\
35.0 & 59.4602547 & $22 / 04 / 2010$ & $16: 10: 33$ \\
40.0 & 62.349662799999997 & $22 / 04 / 2010$ & $16: 10: 40$ \\
45.0 & 64.6050568 & $22 / 04 / 2010$ & $16: 10: 49$ \\
50.0 & 66.988029499999996 & $22 / 04 / 2010$ & $16: 10: 57$ \\
55.0 & 69.321937599999998 & $22 / 04 / 2010$ & $16: 11: 05$ \\
60.0 & 70.059989900000005 & $22 / 04 / 2010$ & $16: 11: 12$ \\
65.0 & 69.4338225999999999 & $22 / 04 / 2010$ & $16: 11: 20$ \\
70.0 & 69.983436600000005 & $22 / 04 / 2010$ & $16: 12: 07$ \\
75.0 & 70.911895799999996 & $22 / 04 / 2010$ & $16: 12: 13$ \\
80.0 & 71.583206200000006 & $22 / 04 / 2010$ & $16: 12: 21$ \\
& & & \\
\hline
\end{tabular}

Fuente: Elaboración propia

\section{INTERPRETACIÓN DE RESULTADOS}

- Se observa que los datos extraídos mediante la sentencia SQL son numéricamente igual a los datos de las Figuras 14 y 16, obedeciendo a la restricción de la apertura de la válvula de la consulta.

- Se corrobora que la interacción entre herramientas SCADA son fiables y proporcionan información valiosa para la toma de decisiones.

- Se confirma que a mayor apertura de la válvula de control proporcional, el caudal será mayor, puesto que al tener una mayor sección transversal bajo las mismas condiciones, aumentará el flujo de líquido a través de dicha válvula.

\section{CONCLUSIONES}

1. La gestión de base de datos es una de las disciplinas que apoyadas por las herramientas de software disponibles en el mercado y las técnicas de modelamiento y diseño hacen que se vuelva imprescindible su empleo en todo tipo de proceso ya sea administrativo o de producción.

2. INTOUCH y LABVIEW son herramientas de software líderes en el mercado conocidos como SCADA, cuya función principal es la supervisión industrial es la supervisión de variables de control, el reconocimiento de imágenes patrón, el registro de datos, el manejo de alarmas y el mantenimiento de parámetros del sistema. 
3. En la industria moderna se hace necesario el trabajo multidisciplinario para el control y supervisión de procesos industriales para gestionar en los distintos niveles de la automatización industrial (nivel de campo, de control de procesos, de supervisión, de diseño y planificación).

4. Debemos estar preparados para la consultoría y actividades propias de nuestra formación como ingenieros industriales para proponer, diseñar y ejecutar soluciones alternativas en el campo de la Automatización Industrial, más aún en este contexto globalizado que nos encontramos y que cada día se hace más evidente.

\section{REFERENCIAS BIBLIOGRÁFICAS}

[1] Acedo, J (2002). Control Avanzado de Procesos. Edit. Diaz de Santos, Madrid, España.

[2] Poma, J., Tello, R., Ruiz, E. (2007). Diseño de una estación virtual para el control de las perturbaciones que afectan la temperatura de los procesos industriales. Industrial Data. Vol 7, N. ${ }^{\circ} 1$ : 33-41

[3] Tello, R. (2003). Bases de Datos en la Ingeniería y los negocios. Industrial Data. Vol 6, N. ${ }^{0} 1$ : 79-82. 